In Situ

Revue des patrimoines
In Situ

Revue des patrimoines

$41 \mid 2019$

Patrimoines gastronomiques. Définitions, typologies et enjeux de conservation

\title{
La recherche du patrimoine végétal vivrier d'hier
}

Looking for the heritage of ancient food plants

Yves-Marie Allain

\section{(2) OpenEdition}

Journals

Édition électronique

URL : http://journals.openedition.org/insitu/25409

DOI : 10.4000/insitu. 25409

ISSN : 1630-7305

Éditeur

Ministère de la Culture

Référence électronique

Yves-Marie Allain, «La recherche du patrimoine végétal vivrier d'hier », In Situ [En ligne], 41 | 2019, mis en ligne le 09 décembre 2019, consulté le 19 décembre 2019. URL : http://journals.openedition.org/ insitu/25409; DOI : 10.4000/insitu.25409

Ce document a été généré automatiquement le 19 décembre 2019.

\section{(c) $($ ) $(9)$}

In Situ Revues des patrimoines est mis à disposition selon les termes de la licence Creative Commons Attribution - Pas d'Utilisation Commerciale - Pas de Modification 4.0 International. 


\title{
La recherche du patrimoine végétal vivrier d'hier
}

\author{
Looking for the heritage of ancient food plants
}

Yves-Marie Allain

\section{Introduction}

1 Les lieux de cultures vivrières échappent aujourd'hui à l'ostracisme que certains courants de l'art des jardins leur avaient imposé et les codes culturels de la séparation des cultures en jardins distincts, - verger, potager, fleuriste, médicinal ou encore de propreté ${ }^{-}$tombent à la fin du $\mathrm{xx}^{\mathrm{e}}$ siècle. Dans certaines villes, les plantes alimentaires remplacent dorénavant, dans les massifs décoratifs, les fleurs devenues le symbole d'un passé révolu ; de nouveaux codes culturels et culturaux s'instaurent, mettant à mal des règlements de lotissements qui interdisaient, il y a encore quelques décennies, la culture des légumes et des arbres fruitiers.

2 Les hiérarchies « sociales » des végétaux furent jusqu'à une période récente une réalité et les mésalliances dénoncées même lorsqu'elles se cachaient derrière les murs des jardins de production.

Rien n'est encore plus fréquent que de trouver des plates-bandes de fleurs le long des allées principales d'un jardin potager, ou de voir des rosiers et autres arbustes de fantaisie alterner avec des arbres fruitiers. C'est là de la floriculture plébéienne, suffisante dans le jardin d'un fermier ou d'un petit propriétaire rural [...]. Dans les châteaux et les maisons de plaisance des personnes riches et aisées, le jardinage fleuriste se dégage ordinairement de ces cultures vulgaires ${ }^{2}$.

Qu'écriraient alors ces messieurs, professeurs du Muséum de Paris, s'ils parcouraient les jardins partagés contemporains qui mêlent allègrement légumes et fleurs, condiments et plantes médicinales?

4 Sans doute faut-il revenir à cette forme de sagesse que préconisait Olivier de Serres, qui considérait qu'une terre bien travaillée ne pouvait rester nue, y compris celle des compartiments de broderie. Les vides et les parties assez larges « serviront à y mettre 
de la potagerie de mesnage : laquelle bordée d'herbes de bonne senteur [...], rendront le jardin de profitable beauté3.»

5 Pour une partie de la société contemporaine, née avec l'industrialisation de l'alimentation et la perte des références aux modes de production et aux cycles ancestraux, le rapport aux légumes et aux fruits se modifie depuis une ou deux décennies, avec un mélange de nostalgie et de volonté de construire un monde différent. Des productions du potager et du verger sortent de l'oubli ${ }^{4}$ en retrouvant leurs lettres de noblesse, favorisées par la création des jardins partagés et promues par de grands chefs de cuisine qui se sont réapproprié ce monde du terroir (fig. 1).

Figure 1

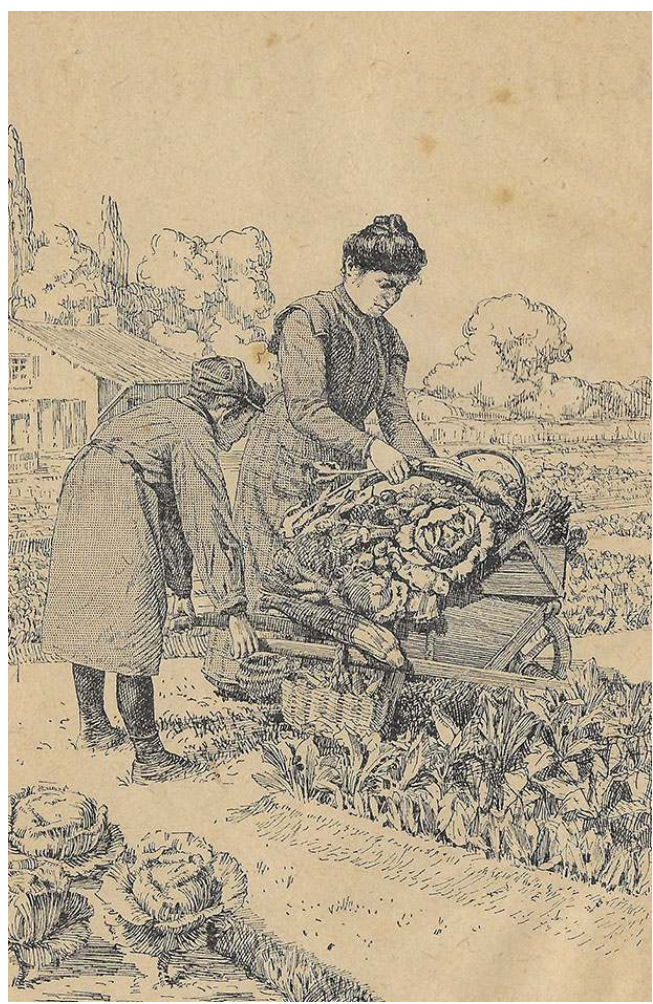

L'approvisionnement au jardin potager. VERCIER, Joseph. Culture potagère. Paris : Hachette, 1926 ; Frontispice.

Mais de quel patrimoine parlons-nous, combien de plantes se trouvent concernées, combien de genres et espèces botaniques sont inclus dans les plantes alimentaires cultivées dans un jardin, que ce soit en France ou dans le monde? Si l'on analyse les listes des plantes alimentaires proposées dans divers ouvrages dont le Bon Jardinier entre 1839 et 1935, le nombre d'entrées pour les potagères est toujours de l'ordre de 100 à 110 , et pour les fruitières entre 18 et 24 . Ces nombres ne préjugent en rien du nombre de variétés décrites qui sont de plus de 1000 , mais qu'il faudrait comparer aux dizaines de milliers réparties sur le territoire français.

7 À la fin $\mathrm{du}$ XIX ${ }^{\mathrm{e}}$ siècle, après de nombreuses expériences dans son jardin de la région parisienne, Auguste Paillieux ${ }^{5}$ pense que 250 nouveaux légumes pourraient être cultivés et utilisés pour l'alimentation des Français. Si l'on excepte le chou chinois, pe-tsaï ${ }^{6}$ ou le crosne, la très grande majorité des légumes décrits est restée "peu connue ou inconnue ». Plus récemment, en ne prenant en compte que les espèces domestiquées 
cultivées dans le monde et utilisées comme légumes, Stanley Kays ${ }^{7}$ retient quelque 400 espèces.

\section{Ambiguiité actuelle du vocabulaire et des références}

Mais ce renouveau des légumes et des fruits n'est pas sans poser un certain nombre de questions et n'est pas exempt d'ambiguïtés, de dérives sémantiques et de concepts détournés. Légumes anciens, légumes tombés dans l'oubli, vieilles variétés, variétés traditionnelles, variétés locales, semences paysannes, goût authentique, sans parler de sauvegarde de la biodiversité, de retour à la naturalité, d'origine naturelle, de sauvegarde de notre patrimoine naturel, etc., autant de mots, d'expressions rarement explicités, dont il est souvent difficile de cerner la réalité qu'ils portent ou qu'on veut bien leur faire porter.

Un mot est remis au goût du jour, celui de «naturalité». Cette recherche de la naturalité ou d'une naturalité est bien celle qui préside au renouveau des légumes et fruits anciens et traditionnels, celle que peut produire un terroir avec des pratiques agronomiques qui respectent les sols et l'environnement.

Ainsi, le chef étoilé Alain Ducasse écrit : « Se nourrir de façon plus saine et naturelle est aujourd'hui une attente et une nécessité qu'il est temps de traduire dans le domaine de la haute cuisine. Des produits exceptionnels s'exprimant dans leur simplicité, une technique qui a l'élégance de s'effacer pour se mettre à leur service. Voilà la cuisine que j'aime sincèrement. Une cuisine de la naturalité libérée, affranchie ${ }^{8}$. " Mais de quelle naturalité est-il question ? Celle de la naturalisation des espèces exogènes qui entrent ainsi dans le patrimoine comme espèces indigènes, grâce aux "lettres de naturalité " de l'Ancien Régime ? À moins que cela ne soit cette naturalité - mot réapparu dans les années 1960 - qui est définie comme un milieu naturel sur lequel la terre et sa communauté de vie ne sont point entravées par l'homme, où l'homme lui-même n'est qu'un visiteur de passage. Mais nous sommes bien loin du potager ou du verger dans lesquels rien de ce qui est produit n'existerait sans la présence et l'action de l'homme (fig. 2). 


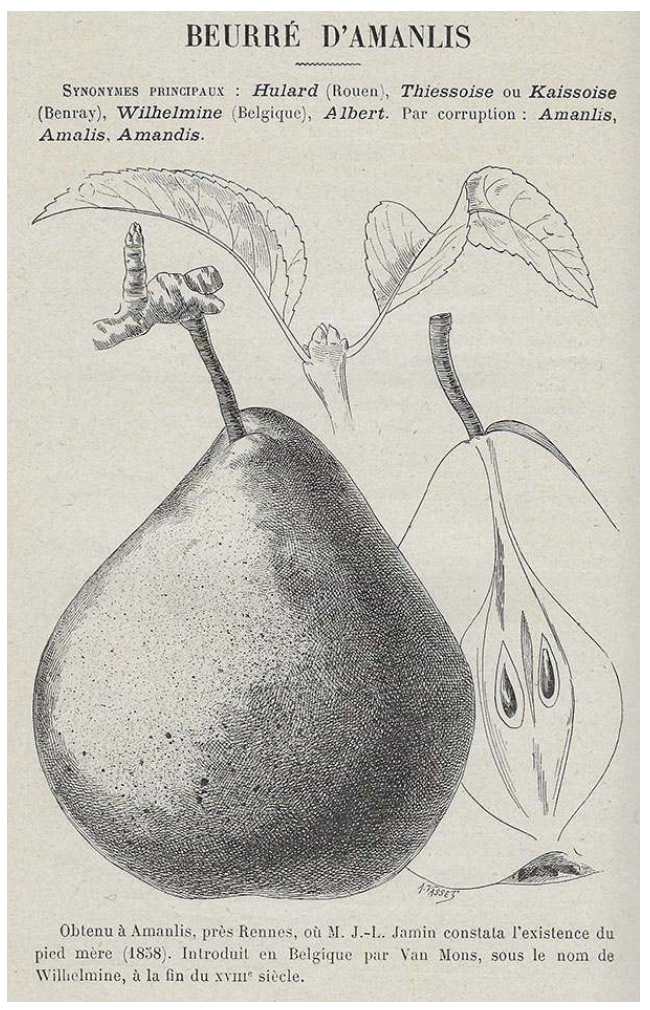

Poire « Beurré d'Amanlis » et la diversité de ses noms. Société nationale d'horticulture de France. Les Meilleurs Fruits au début du xxe siècle. Nouvelle édition revue, au siège de la société, Paris : 1928, p. 356.aud.

Que penser de l'emploi détourné et abusif par certains communicants de l'expression récente de "diversité biologique $»^{9}$ ou "biodiversité " qui ne correspond le plus souvent qu'à l'ombre d'elle-même, l'expression étant employée le plus souvent pour évoquer la diversité végétale, celle des espèces ou des variétés.

Que ce soit au potager ou au verger, les questions de l'origine initiale, indigène ou exotique, des plantes alimentaires et de leurs usages ne sont pas posées, alors que ces mêmes problématiques font conflit en paysage et art des jardins ${ }^{10}$. Si le patrimoine naturel d'un territoire est définissable, comment peut se définir le patrimoine des plantes alimentaires, sachant que dans bien des cas leur domestication remonte à des millénaires et qu'en Europe occidentale, la très grande majorité des plantes furent introduites au fil des siècles. De la même façon, la période à prendre en compte pour la patrimonialisation est-elle celle des introductions ou celle de l'acceptation par les diverses classes de la société (par exemple, pour la pomme de terre, la tomate ${ }^{11}$, etc.).

Mais n'est-ce pas vain à la fois de poser la question et d'en chercher des réponses, car dans le cas des plantes alimentaires, quel est le véritable patrimoine: un nom scientifique et une pléiade de noms vernaculaires, un patrimoine génétique non défini, évolutif, s'adaptant de façon permanente au fil des années aux conditions pédologiques et climatiques, un patrimoine culturel lié à des méthodes culturales et des savoir-faire de jardiniers, acceptés par un groupe social?

Une autre constatation ou contestation revient régulièrement, la perte de la qualité gustative des légumes et des fruits depuis plusieurs décennies. Au début du $\mathrm{xx}^{\mathrm{e}}$ siècle, certains auteurs rapportent les essais comparatifs effectués dans les potagers entre 
divers engrais minéraux et organiques. Ils ont une influence sur la saveur et engendrent des produits qui «ne sont pas absolument identiques tant du point de vue de la composition chimique qu'au point de vue de leur saveur ». «Les nuances de goût, sensibles au palais, proviennent en effet des propriétés physico-chimiques du sol producteur $^{12}$ ». Mais déjà au XVII siècle, La Quintinie, créateur et responsable du Potager du roi à Versailles, remarquait : « [...] combien d'autres y en a-t-il qui se font un plaisir extrême de faire manger des salades et des herbes de leurs jardins, soutenant hardiment qu'elles sont beaucoup meilleures que celles des marchés et des jardiniers ordinaires $[. . .]^{13} »$. Ce que l'on produit soi-même a-t-il toujours un meilleur goût et une meilleure saveur que ce que l'on peut acheter sur un marché?

15 Tous ces mots et expressions ne sont pas sans poser de nombreuses questions quant à la réalité recherchée, à la possibilité réelle de retrouver un patrimoine végétal très largement anthropisé et ce, parfois depuis des millénaires ${ }^{14}$.

\section{Le travail de collectage et de recensement}

16 Au cours du néolithique, l'homme prend conscience qu'il est possible de maitriser certains éléments vivants à son profit. Ainsi, pendant des millénaires, le jardinier va façonner des variétés locales par une sélection en grande partie inconsciente et naturelle, sous l'influence de l'environnement. Des ethnologues et philosophes pensent que le jardinier effectue une "action de pilotage", et non une "action technique " comme l'art de l'artisan. Il influe sur les processus naturels et sur les végétaux et grâce à un "empirisme attentif», il en modifie progressivement les caractéristiques et qualités par rapport à leur état sauvage.

17 La rupture dans ces méthodes de sélection apparaît au cours du XIX ${ }^{e}$ siècle, lorsque des sélectionneurs vont souhaiter améliorer les variétés locales de façon délibérée et systématique. "Ce processus a permis l'émergence d'une série de variétés plus évoluées, appelées cultivars. Il a abouti aux cultivars modernes à haut rendement, souvent appelées cultivars d'élite ${ }^{15}$. $\gg$ Ce dernier mot fut largement diffusé par le slogan des établissements Clause avec leurs "graines d'élite Clause». Ces cultivars, qui correspondent en grande majorité aux variétés dites anciennes, ont progressivement remplacé, chez les professionnels puis chez les amateurs, les variétés locales, introduisant par ce biais une forme d'uniformité génétique (fig. 3). 


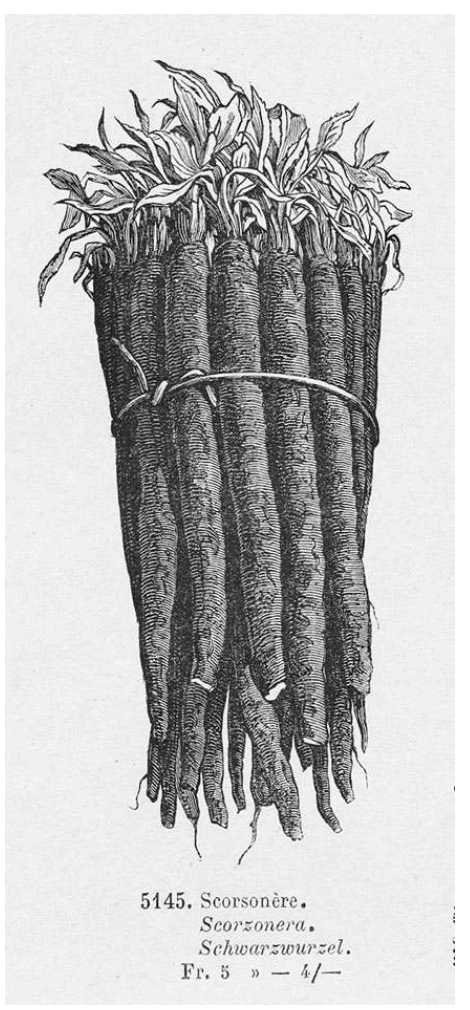

Scorsonere. Album de clichés électrotypes, comprenant toutes les illustrations des ouvrages et catalogues publiés par Vilmorin-Andrieux \& $C^{i e} .2^{\mathrm{e}}$ éd. Paris : Vilmorin-Andrieux \& $C^{\mathrm{ie}}, 1888$, plantes potagères, p. 259. statut de « locale » ou de «traditionnelle»? La plupart des variétés le sont devenues parce qu'elles ont été adoptées, un jour ou l'autre, par les habitants d'un terroir. Ces variétés convenaient à la fois aux conditions climatiques, au sol, aux possibilités des techniques culturales, et surtout aux besoins d'une population. Il ne faut pas sousestimer les aspects tant sociaux que culturels dans la gestion des plantes, en particulier dans les systèmes de diffusion, d'échange, de transmission des graines et semences, des greffons. Voilà pourquoi il n'est pas rare de rencontrer des variétés étrangères au terroir ou même à la France, qui ont été " assimilées » localement. «En effet, leur culture a laissé des traces, témoins de leur rôle dans l'économie régionale et dans la mémoire collective. Cela tend à prouver que le statut de local ou de traditionnel dépend moins de la provenance d'un cultivar que de l'importance qu'il a acquise sur place au fil des décennies ${ }^{16}$. » Pour ces raisons, la variété ou le cultivar local «perd tout son sens s'il est séparé de son contexte global, écologique et ethnologique ».

Dès la fin des années 1960, grâce au travail de recensement de passionnés, professionnels ou amateurs, de collectionneurs avertis et défenseurs du patrimoine cultural et culturel régional et local, il fut possible de créer des jardins et vergers conservatoires, des centres de documentation ethnologique, de publier des fascicules faisant le point des connaissances sur les variétés anciennes et locales ${ }^{17}$, de monter des expositions ${ }^{18}$. Parmi les organismes fédérateurs qui jouèrent un rôle non négligeable, il faut citer l'Association française pour la conservation des espèces végétales (AFCEV) fondée en 1983 à l'initiative du ministère en charge de l'Écologie, et le BRG - Bureau des ressources génétiques - créé également en 1983 à l'initiative des ministères en charge 
de la Recherche, de l'Agriculture, de l'Écologie. Ils ont largement aidé à rationaliser la collecte des données, les études et leur diffusion et surtout, à faire reconnaitre, y compris auprès des pouvoirs publics, la valeur patrimoniale, culturale et culturelle des variétés locales et anciennes ${ }^{19}$ (fig. 4).

Figure 4

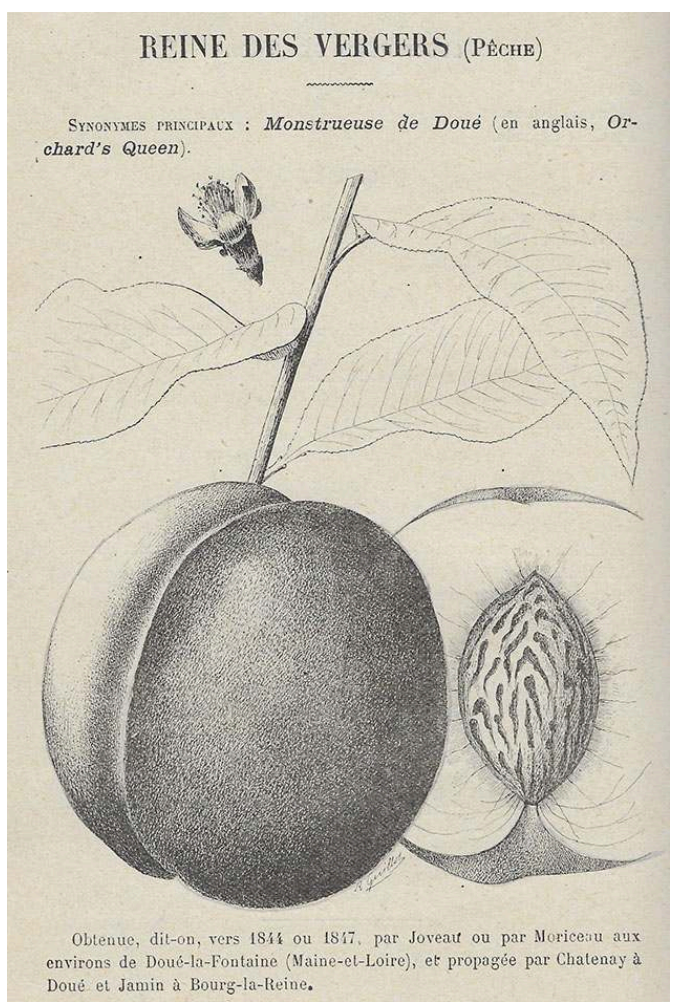

Pêche "Reine des vergers », ou la difficulté pour retrouver une origine. Les Meilleurs Fruits au début du xxe siècle, p. 318.

Le patrimoine des vergers fut plus aisé à inventorier car les végétaux vivent plusieurs décennies. Le travail de détermination, de classement, de mise en ordre des dénominations locales, pour le moins hétérogènes, a permis de sauvegarder in situ et dans les vergers conservatoires les variétés locales et anciennes. Pour les légumes, ce travail de connaissance est beaucoup plus délicat dès lors que les plantes sont à cycle court, que les semis sont souvent obligatoires tous les ans ou tous les deux ans, que l'origine de nos légumes est surtout exogène.

21 Les scientifiques estiment que les variétés locales traditionnelles sont des populations de plantes que Roger de Vilmorin ${ }^{20}$ dénommait «cultivars-populations» et les généticiens contemporains "variétés-populations». Elles ne sont proches qu'en apparence, présentent une diversité génétique certaine et possèdent de ce fait un pouvoir évolutif qui leur permet de s'adapter en continu aux variations du milieu.

Malgré un long travail de collectage et de rationalisation des dénominations, une approche nouvelle de la génétique, le patrimoine végétal des légumes et des fruits garde une grande partie de son ambiguïté grâce à sa diversité tant biologique, ethnologique que sociale (fig. 5). 


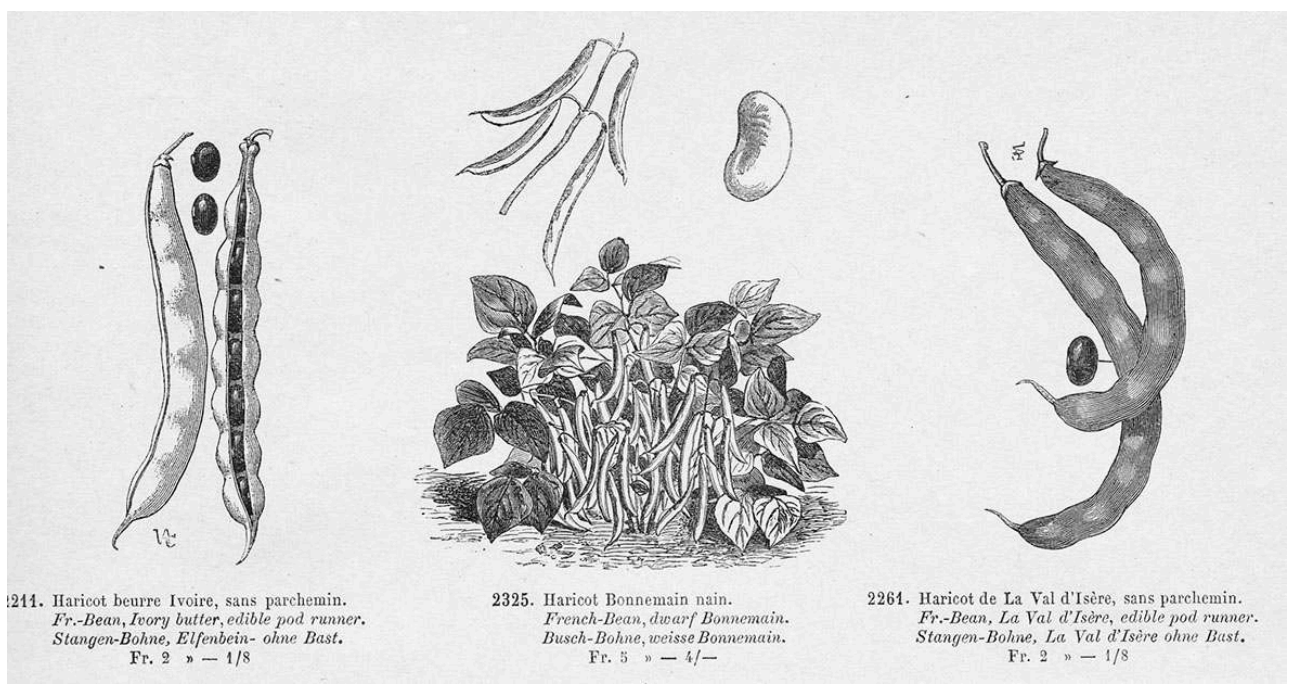

Quelques variétés de haricots. Album de clichés, plantes potagères, p. 111.

\section{Les pratiques des jardiniers}

Au potager comme au verger, le personnage primordial est et reste le jardinier avec ses méthodes de travail et son approche de son rôle et de sa mission.

$\mathrm{Au}$ début $\mathrm{du} \mathrm{xx}^{\mathrm{e}}$ siècle, dans son ouvrage sur la culture potagère, Joseph Vercier ${ }^{21}$ détermine quatre types de lieux de cultures : le jardin potager ou potager, celui réservé à la «culture de tous les légumes nécessaires à un ménage », le jardin maraîcher avec une " production ininterrompue ou commerciale de légumes ", la « culture maraîchère champêtre » ou culture de quelques légumes en plein champ (choux, navets, oignons, asperges, etc.), enfin, le jardin mixte ou potager-fruitier dans lequel se cultivent à la fois des légumes et des arbres fruitiers. Sans remonter à la fin du Moyen Âge, ce classement confirme que, depuis plus d'un siècle, les impératifs de production des divers acteurs ne sont pas les mêmes et que l'origine des variétés peut être hétérogène, depuis les marchands grainiers jusqu'aux variétés locales. Il ne faut pas sous-estimer les apports techniques dont les serres, les engrais, les produits issus de l'industrie chimique pour lutter contre les maladies et prédateurs, le rôle des maisons grainières et de leur réseau, des sociétés d'horticulture, des publications, des foires et des concours du plus gros légume, du plus beau fruit, etc. L'ensemble de ces nouveautés va progressivement perturber et faire perdre la filiation d'une philosophie de travail issue des jardiniers des siècles précédents et modifier le rapport au jardin.

Certaines des recommandations faites aux jardiniers ne facilitent pas la permanence des variétés locales: «Vous pouvez prétendre récolter vous-mêmes quelques graines dans votre jardin, à la condition de ne faire que les plus courants (radis, oignons, salades, etc.) et de les renouveler tous les deux à trois ans par des produits du commerce 22 ", ou alors, en prenant de grandes précautions pour éviter les hybridations entre variétés de haricots et "pour les conserver franches, il faut absolument les cultiver le plus loin possible les unes des autres ${ }^{23}$ ». Mais les habitudes d'une autoproduction de ses graines semblent être encore la règle dans certaines provinces comme cela est rapporté dans un opuscule de 1998 sur les jardins de Basse-Normandie. 
Il y est précisé qu' ' il y a 50 ans, la plupart des jardiniers récoltaient leurs graines d'une année sur l'autre. Ils suivaient là les principes de la littérature horticole diffusés dans les écoles primaires rurales depuis la fin du siècle dernier ${ }^{24}$ » (fig. 6, fig. 7).

\section{Figure 6}

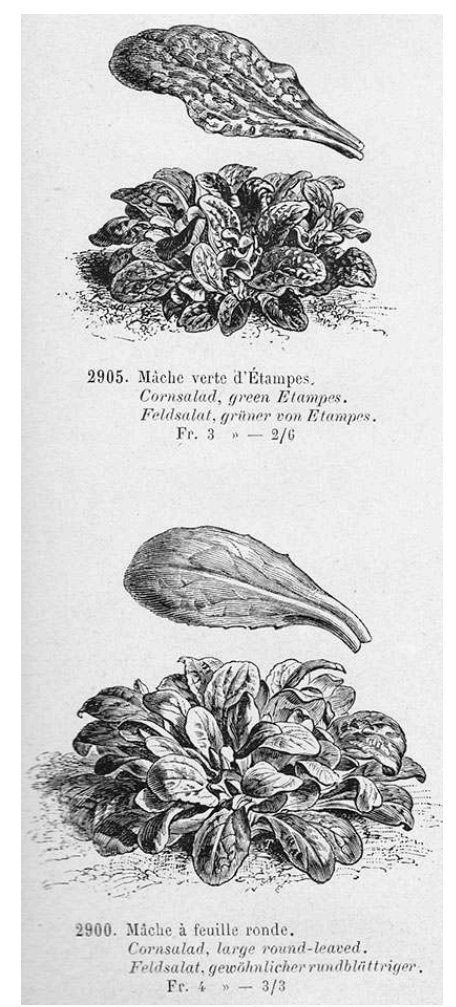

La diversité des mâches. Album de clichés, plantes potagères, p. 135. 
Figure 7

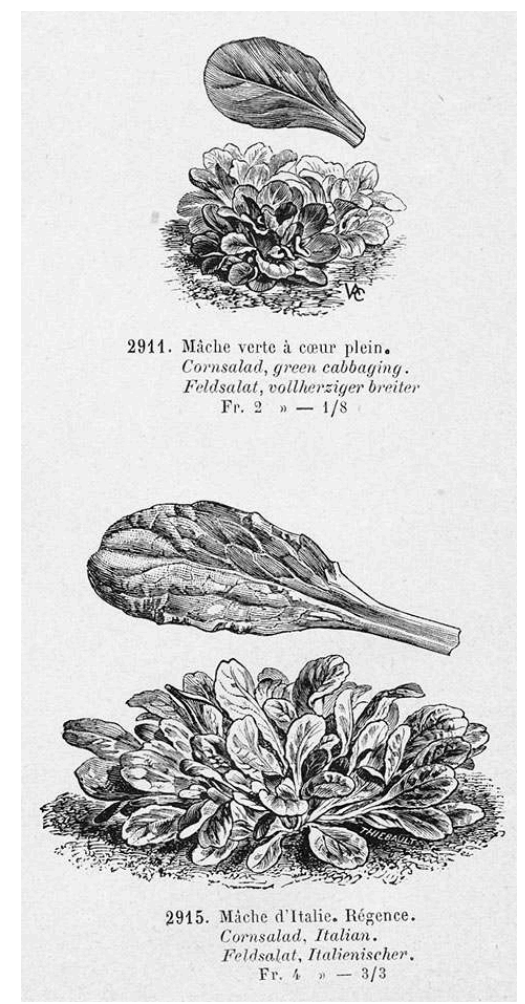

La diversité des mâches. Album de clichés, plantes potagères, p. 135.

Comment espérer s'y retrouver aujourd'hui quand, dès la seconde moitié du XIX siècle, des doutes existent sur l'origine d'une variété : «L'asperge d'Argenteuil elle-même paraît être une variété de l'asperge de Hollande améliorée par la culture ${ }^{25}$. Mais alors, comment interpréter l'origine des autres variétés citées de l'asperge de Hollande: violette de Vendôme, de Besançon, d'Ulm, de Marchiennes?

Le Bon jardinier de 1864 cite l'expérience ayant pour objectif l'étude de l'amélioration et de la transformation de la carotte sauvage en plante alimentaire. L'auteur rapporte qu'en trois générations, les carottes obtenues ont l'apparence des carottes du jardin avec « une chair un peu plus compacte, leur saveur plus douce; elles ont été trouvées supérieures aux anciennes variétés». Au fur et à mesure des semis, il a «vu successivement sortir de cette souche presque toutes nos anciennes variétés ${ }^{26}$ », avec les diverses couleurs des racines, depuis les blanches jusqu'aux rouges en passant par les jaunes et les lie-de-vin. Cette expérience montre qu'on ne peut exclure l'influence de la plante sauvage sur les diverses générations de celle cultivée. Combien de variétés locales se sont ainsi hybridées au cours des siècles avec leurs ancêtres indigènes, modifiant involontairement les caractères de la plante cultivée ? (fig. 8) 


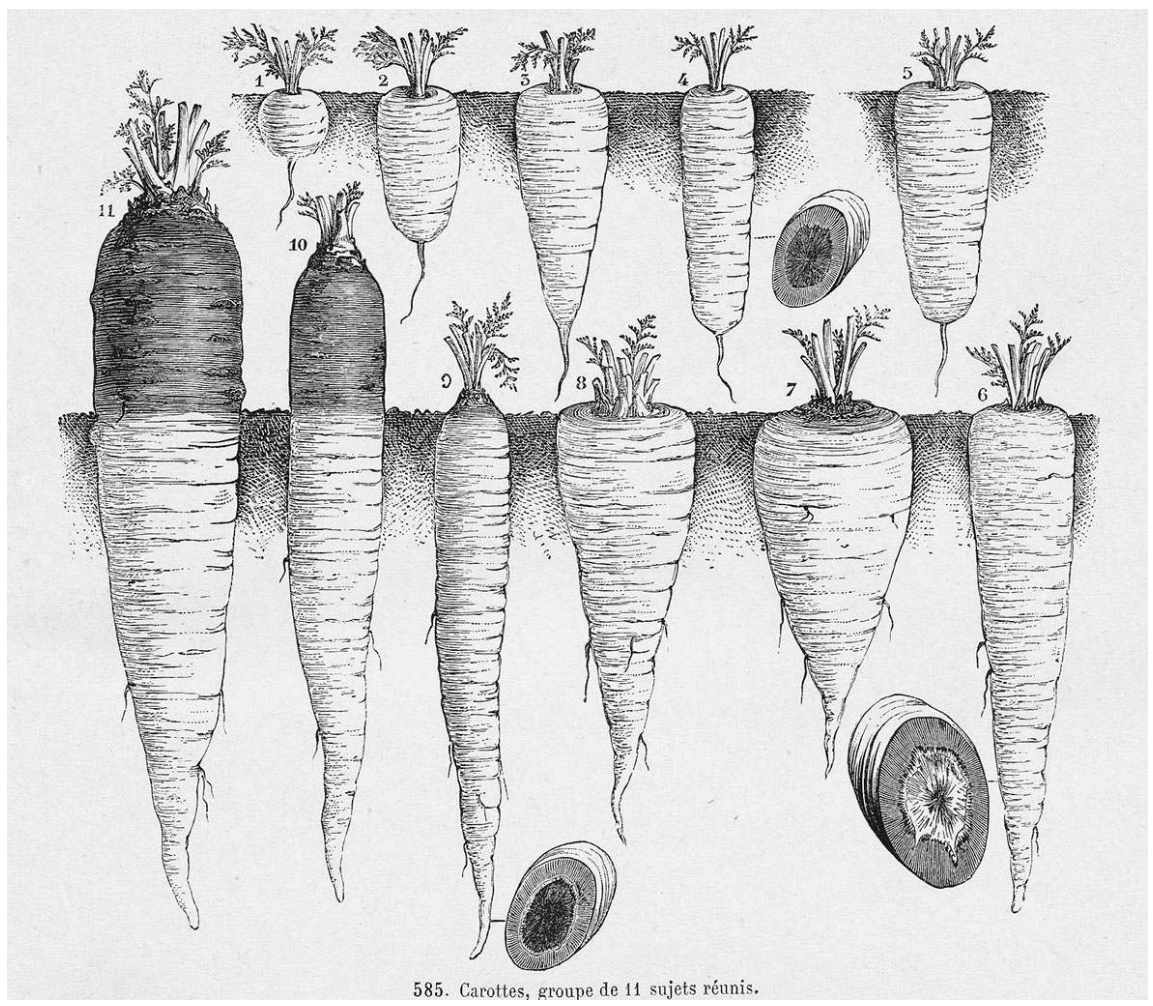

Les diverses formes des racines de carotte. Album de clichés, plantes potagères, p. 33.

La maison grainière Vilmorin, en 1888, publie un album de clichés ${ }^{27}$ présentant les multiples variétés proposées à ses clients, que ce soit en légumes, fleurs et autres végétaux ornementaux. Pour la partie légumière et condimentaire, plus de 1600 variétés et formes sont proposées pour une centaine d'entrées par les noms français.

Aucune information ne figure sur le nombre de variétés, de formes, etc. qui a été testé, comment cette sélection a été opérée, ni quel fut le travail d'amélioration effectué pour choisir les variétés considérées comme commerciales et suffisamment stables pour les inscrire au catalogue, par exemple, 57 variétés de chou, 68 de pois, 34 de navets, etc. Mais cette stabilité n'est pas toujours parfaite car on trouve deux clichés avec des formes différentes pour le navet long des Vertus pointu et trois clichés pour le navet long de Briollay, ou pour celui de Saint-Brieuc !

L'imprécision se trouve renforcée pour certains des noms donnés à ces cultivarspopulations. Ils sont issus soit des noms de la variété locale initiale, soit des lieux où furent prélevées les graines. On trouve la carotte rouge : demi-longue nantaise, de Luc, de Carentan - demi-courte obtuse de Guérande - longue lisse de Meaux - longue de Saint-Valéry, d'Altringham; la carotte blanche: des Vosges, améliorée d'Orthe. En prenant une autre plante, l'oignon, sont cités : l'oignon blanc : de la Reine, de Hollande, de Paris, de Valence, de Nocera; l'oignon jaune: d'Italie, de Côme, de Danvers, d'Espagne, de Zittau, de Saint-Laurent, de Trébons, de Russie ; l'oignon rouge : de Niort, de Rocca, de Madère ou de Tripoli, de Mézières, d'Italie ; enfin l'oignon d'Égypte, de Mulhouse, l'oignon Catawissa (fig. 9). 


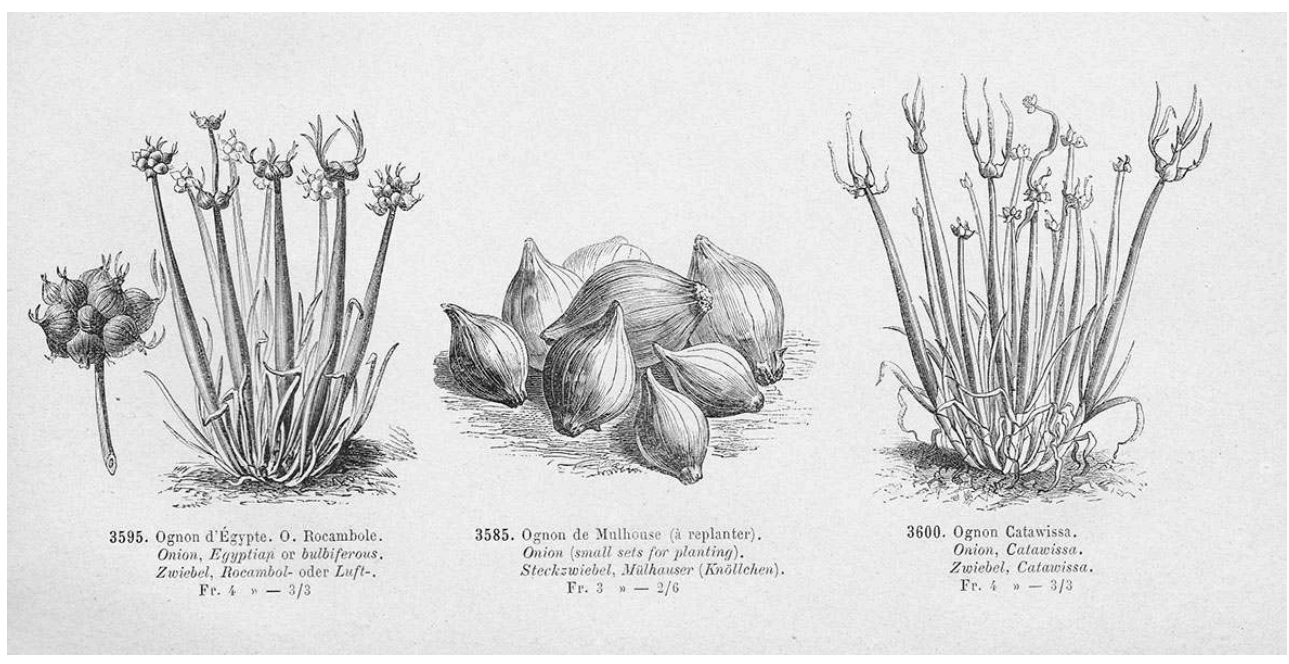

Quelques espèces et variétés d'oignons comestibles. Album de clichés, plantes potagères, p. 167.

$31 \mathrm{Au}$ verger, l'origine est souvent imprécise et la dénomination d'une même variété, multiple. Dès le début du XIX ${ }^{e}$ siècle, "un grand mouvement se dessine dans les échanges entre localité et entre pays; de plus, il est fait de nombreux semis, dont les résultats sont étudiés par la greffe ${ }^{28} »$.

La mention "origine inconnue", "origine ancienne et inconnue», "cette variété semble avoir été trouvée dans... » suivie du nom de la région concernée, figure pour plusieurs dizaines de variétés de fruits divers avec parfois des commentaires qui montrent toute la difficulté à remonter le temps comme pour la variété de cerise griotte du Nord: «certains auteurs la prétendent originaire de Hollande, d'autres de Russie »!

Ces quelques exemples montrent l'ampleur des difficultés actuelles et rendent encore plus délicate la différenciation entre une variété locale et une variété ancienne, entre une variété issue du seul travail d'une communauté villageoise et un cultivar réintroduit.

\section{De grands absents : terre végétale et climat}

Il n'est pas possible de parler de légumes, de fruits donc d'arbres fruitiers, sans aborder l'élément primordial et indispensable qu'est la terre végétale. Support physique, chimique, biologique, son rôle a été largement sous-estimé. Il n'existe pas de véritable définition, encore moins de protection réglementaire. Contrairement aux plantes, aux animaux, aux habitats, à l'eau, le sol, support de la vie, ne fait l'objet d'aucune convention internationale, et la directive ${ }^{29}$ prévue par l'Europe a même été retirée faute d'accord.

Cette directive thématique, avortée, indiquait clairement dans son préambule que le sol, couche supérieure de la croûte terrestre,

est constitué de particules minérales, de matières organiques, d'eau, d'air et d'organismes vivants. Le sol est l'interface entre la terre, l'air et l'eau et abrite la majeure partie de la biosphère. La formation des sols étant un processus extrêmement lent, on peut considérer qu'il s'agit essentiellement d'une ressource 
non renouvelable. [...] Toute dégradation de sa structure détériore également les autres milieux naturels et écosystèmes. France jusqu'au milieu du $\mathrm{xx}^{\mathrm{e}}$ siècle ont été progressivement remplacées par des cultivars plus homogènes, plus productifs, obtenus par les sélectionneurs professionnels. Mais depuis quelques décennies, les variétés-populations de céréales, de plantes alimentaires, fourragères, textiles, reviennent sur le devant de la scène ${ }^{31}$. Des traités comme celui de la $\mathrm{FAO}^{32}$ de $2001^{33}$, des directives européennes ${ }^{34}$ rendent possibles «certaines dérogations pour l'admission des races primitives et variétés agricoles naturellement adaptées aux conditions locales et régionales et menacées d'érosion génétique, [...] ». Le Catalogue officiel français des espèces et variétés de plantes cultivées ${ }^{35}$ possède pour les espèces légumières une liste " $\mathrm{c}$ » sur laquelle peuvent être inscrites les variétés de conservation avec des critères biologiques et géographiques restrictifs : « races primitives et variétés de légumes traditionnellement cultivées dans des localités et régions spécifiques et menacées d'érosion génétique.» $\mathrm{Si}$, sous la pression de différentes associations, les réglementations autour des variétés anciennes ou locales se sont assouplies ${ }^{36}$, cela ne résout pas l'ensemble des problèmes liés à la conservation, à la transmission, à la mise en culture, à la diffusion, au suivi d'un patrimoine génétique par nature divers, car toujours porteur de la part de naturalité des millénaires passés.

En dépit des contraintes réglementaires et administratives, toutes les enquêtes, tous les conservatoires, toutes les publications à caractère ethnologique, toutes les volontés de vulgarisation de variétés légumières et fruitières d'autrefois n'auront de sens et d'avenir que si est mise en œuvre une politique dynamique de constitution d'un 
nouveau patrimoine, par nature aléatoire, issu de méthodes respectueuses de la dynamique sélective naturelle dont celles issues de l'épigénétique ${ }^{37}$.

Dans notre société, chaque jardinier qui, selon Karel Čapek, est « indubitablement un produit de la civilisation et pas du tout de l'évolution naturelle ${ }^{38}$ » se doit de relever un défi, celui de favoriser l'émergence de nouvelles variétés-populations locales adaptées aux conditions pédologiques et climatiques contemporaines de chaque terroir. La redécouverte de ce travail de sélection issu de méthodes ancestrales doit s'effectuer à partir des variétés-populations et cultivars-populations qui peuvent encore exister sur place. Une variété, bien qu'avant tout être biologique, est également un produit culturel qui doit être accepté et répondre aux attentes multiformes d'une société en devenir. Cette soumission nouvelle aux cycles de la nature, jusqu'à en devenir dépendant, à la rotation aléatoire des légumes et des fruits, à leurs disponibilités incertaines, est saine, positive, porteuse d'un espoir, et elle nous réintégrera comme membre à part entière de la communauté des êtres vivants (fig. 10).

Figure 10

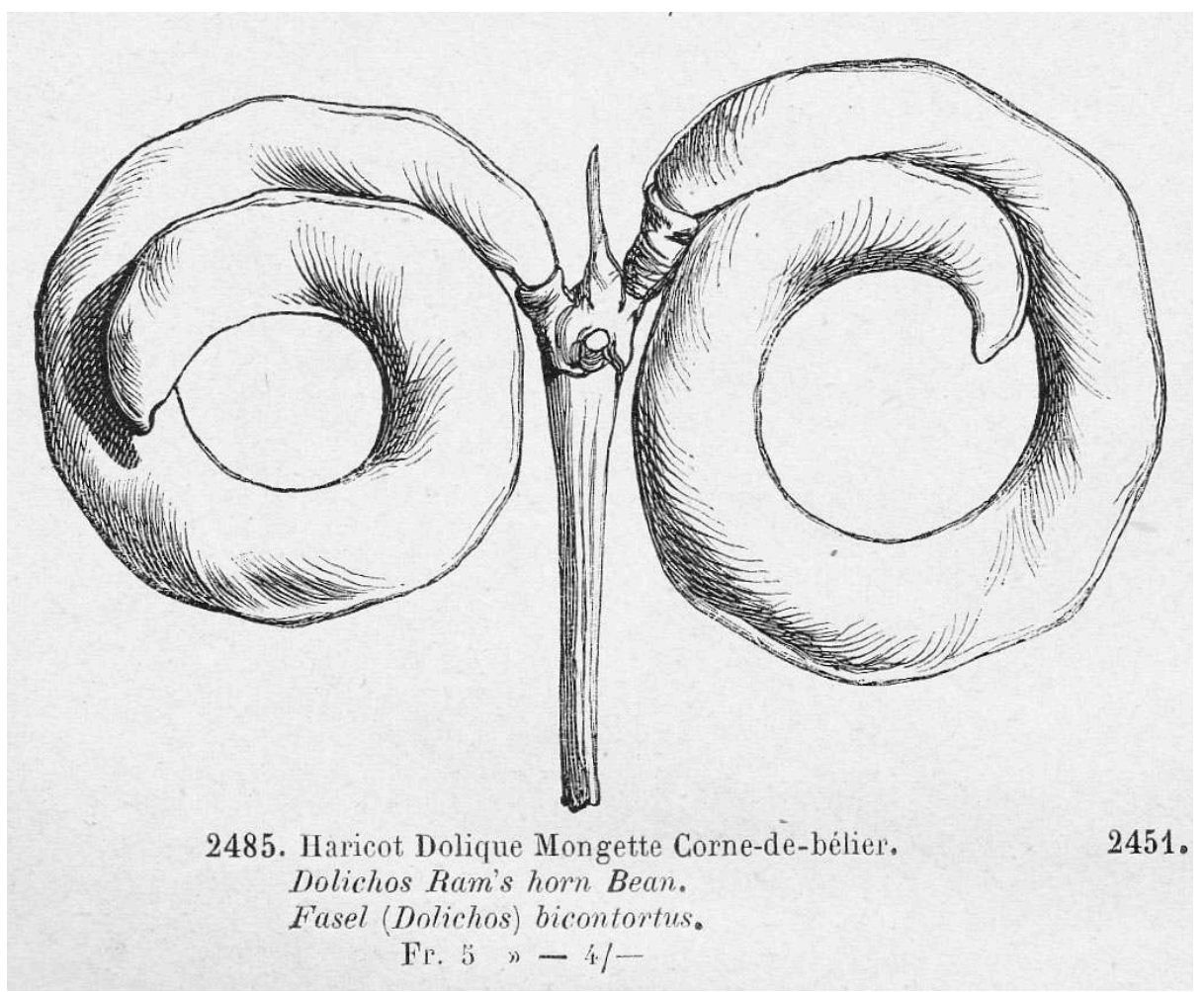

Une variété de haricot : « haricot dolique mongette corne-de-bélier ». Album de clichés, plantes potagères, p. 121. 


\section{NOTES}

1. - À partir du milieu du XVII ${ }^{\mathrm{e}}$ siècle, il s'agit du jardin qui donne un air de propreté à la demeure auprès de laquelle il est dressé. Il portera ultérieurement le nom de jardin d'agrément.

2. - DECAISNE, Joseph, NAUDIN, Charles. Manuel de l'amateur des jardins. Traité général d'horticulture. Paris : Firmin Didot Frères, [1862-1871].

3. - SERRES, Olivier de. Le Théâtre d'agriculture et mesnage des champ [1600]. Grenoble: Dardelet, 1973, t. II, p. 100.

4. - PAILlIEUX, Auguste, BOIS, Désiré. Le Potager d'un curieux, histoire, culture \& usages de 250 plantes comestibles peu connues ou inconnues [1885] $3^{\mathrm{e}}$ édit. Paris : Librairie agricole de la maison rustique, 1899.

5. - Ibid.

6. - Brassica rapa Pekinensis.

7. - KAYS, Stanley J. Cultivated vegetables of the world: a multilingual onomasticon. Wageningen: Wageningen Academic Publishers, 2011.

8. - Voir le site: https://www.alainducasse-plazaathenee.com/fr/naturalite [consulté le 16/10/2019].

9. - Diversité biologique ou biodiversité : concept scientifique défini dans les années 1980 qui englobe des notions complexes, encore mal connues, non dissociables, incluant la diversité génétique d'une espèce, la diversité des espèces et la diversité des habitats naturels.

10. - Voir ALLAIN, Yves-Marie. Les Plantes exotiques, une réputation perdue? Saint-Nazaire : Petit Génie, 2014.

11. - Pour ces deux plantes, plus de trois siècles se sont écoulés entre leur introduction en Europe (début $\mathrm{XVI}^{\mathrm{e}}$ siècle) et leur emploi alimentaire par toutes les couches de la société.

12. - VERCIER, Joseph. Culture potagère [1911]. 10 éd. Paris : Hachette, 1926, p. 61.

13. - LA QUINTINIE, Jean-Baptiste de. Instructions pour les jardins fruitiers et potagers. Paris : Barbin, 1690, Sixième partie, De la culture des potagers.

14. - Voir LA BOUËRE, Mériadec de, ill. MAHER, Marie. Légumes et fruits, quelle naturalité aujourd'hui ? Le rôle des saisons, des cycles et des rythmes biologiques. Saint-Nazaire : Petit Génie, 2017, p. 7.

15. - HOYT, Erich. La Conservation des plantes sauvages apparentées aux plantes cultivées. Trad. Pierre Roche, Michel Chauvet et André Charrier. Paris : Bureau des ressources génétiques, 1992, p. 9.

16. - MARCHENAY, Philippe, avec la collab. de LAGARDE, Marie-France. À la recherche des variétés locales de plantes cultivée : guide méthodologique. Porquerolles : PAGE-PACA, 1987.

17. - Voir LETERME, Évelyne, OLIVIER, Louis. « Les Collections fruitières de variétés anciennes et locales en France [...] ». Sauve qui peut ! $\mathrm{n}^{\circ}$ 3, 1992, p. 23-28.

18. - «Légumes et fruits, du Jardin du Roy au Jardin des plantes », Paris, Jardin des plantes, 25 mars-14 septembre 1992 ; "Cueillettes sauvages ???", Genève, Conservatoire et Jardin botanique, 1993 ; « Légumes d'hier et d'aujourd'hui, gestes et saveurs à redécouvrir », château de Namur (Belgique), 4 juillet-2 septembre 2001.

19. - Voir ACFEV/BRG. Un Patrimoine : les variétés locales d'espèces fruitières. Actes du symposium de Nancy, 6-8 septembre 1984. Journal d'agriculture traditionnelle et de botanique appliquée, 1985.

20. - Roger Lévêque de Vilmorin (1905-1980), généticien et horticulteur français, membre de l'Académie d'agriculture.

21. - VERCIER, Joseph. Op. cit.

22. - Ibid., p. 97.

23. - Le Nouveau Jardinier illustré. Paris : E. Donnaud, année 1875, t. I, p. 508.

24. - VIVIER, Michel. Jardins ruraux en Basse-Normandie. Caen : C.Ré.C.E.T., 1998, p. 16. 
25. - Le Nouveau Jardinier illustré. Op. cit., t. I, p. 441.

26. - Le Bon Jardinier. Almanach horticole pour l'année 1864. Paris : Librairie agricole de la Maison rustique, 1864, p. 437.

27. - Album de clichés électrotypes, comprenant toutes les illustrations des ouvrages et catalogues publiés par Vilmorin-Andrieux \& $C^{i e}$. Paris : édit. Vilmorin-Andrieux \& $C^{\mathrm{ie}}, 1888$.

28. - Société nationale d'horticulture de France. Les Meilleurs Fruits au début du $X x^{e}$ siècle. Nouvelle édition revue, au siège de la société. Paris : 1928, p. 10.

29. - Décision $n^{\circ} 1600 / 2002 / C E$ du Parlement européen et du Conseil du 22 juillet 2002 établissant le sixième programme d'action communautaire pour l'environnement (JO L 242 du 10 septembre 2002, p.1). En mai 2014, la directive européenne en faveur de la protection des sols est officiellement retirée par la Commission européenne, faute de consensus entre les divers pays.

30. - Agriculture urbaine: à Paris, alliance inédite entre quatre grands chefs et une coopérative. AFP, 25 octobre 2018 à $14 \mathrm{~h} 30$, mis à jour le 25 octobre 2018 à $14 \mathrm{~h} 30$.

31. - Voir le site: http://www.inra.fr/Chercheurs-etudiants/Systemes-agricoles/Tous-lesdossiers/Selection-classique-ou-participative-plusieurs-strategies-pour-les-bles-bios/Varietespopulations-privilegier-l-adaptabilite/(key)/1 [consulté le 16/10/2019].

32. - Organisation des Nations unies pour l'alimentation et l'agriculture (Food and Agriculture Organization of the UN).

33. - Il s'agit du Traité international sur les ressources phytogénétiques pour l'alimentation et l'agriculture, FAO, 2001, qui, reconnaissant les droits des paysans, le rôle des paysans dans la conservation de la biodiversité, contraint les pays signataires à adopter des actions, des politiques et des programmes pour la conservation in situ et la phytosélection participative.

34. - Voir : Commission européenne, directive 2008/62/CE du 20 juin 2008, directive 2009/145/CE du 26 novembre 2009.

35. - Ce catalogue officiel français (190 espèces, 9000 variétés et cultivars) est établi sous la responsabilité du ministère en charge de l'Agriculture, l'inscription ou la radiation se faisant sur proposition du Comité technique permanent de la sélection des plantes cultivées (CTPS).

36. - La mise en place de ces possibilités n'est pas aussi simple que cela pourrait paraître : voir l'avis (25 octobre 2018) du Conseil constitutionnel censurant l'article 78 de la loi issue des États généraux de l'alimentation qui permettait de vendre aux amateurs des semences sans aucune information garantie et aucun contrôle notamment sanitaire (communiqué de presse du Groupement national interprofessionnel des semences et plants (GNIS), 30 octobre 2018).

37. - L'épigénétique est l'étude des changements d'activité des gènes. L'environnement (sol, air, stress hydrique, etc.) joue un rôle et se trouve à l'origine de variations adaptatives, qui peuvent conduire à des modifications dans l'expression des gènes mais sans en modifier leur séquence d'ADN. Les modifications épigénétiques peuvent être réversibles mais certaines deviennent pérennes, persistent lorsque le signal qui les a induites disparaît et peuvent être transmises d'une génération à l'autre.

38. - ČAPEK, Karel. L'Année du jardinier. Trad. française Joseph Gagnaire. [1933]. Paris : 10-18, 2000, p. 52 . 


\section{RÉSUMÉS}

Les codes culturels de la séparation des cultures en jardins distincts - verger, potager, fleuriste tombent à la fin du $\mathrm{xx}^{\mathrm{e}}$ siècle. La volonté de réhabilitation des variétés anciennes de fruits et de légumes, par une société urbaine, n'est pas exempte d'ambiguïtés, de dérives sémantiques et de concepts détournés. $\mathrm{Si}$, durant des siècles, une continuité certaine des semences et des variétés a prévalu dans chaque potager et verger, à partir $d u$ XIX ${ }^{\mathrm{e}}$ siècle, une rupture de filiation se marque progressivement avec l'apparition des maisons grainières. Celles-ci proposent de nouveaux cultivars issus d'une recherche scientifique et agronomique dédiée à l'amélioration de la production. Retrouver les odeurs, les goûts et les saveurs d'antan, résultat d'une alchimie fort complexe associant le respect du sol et des cycles climatiques, retrouver graines et semences perdues dans un monde vivant qui n'a d'existence que dans une dynamique permanente d'adaptation, de changement, voire d'instabilité, ne relèvent-ils pas d'une vaine recherche?

The cultural codes dictating that plant cultivation should be separated into distinct gardensorchards, vegetable gardens, flower gardens-tended to disappear at the end of the twentieth century. But the desire to rehabilitate old varieties of fruits and vegetables which has emerged within our urban society is not without its ambiguities, semantic shifts and conceptual twists. For centuries a certain continuity prevailed in the seeds and varieties grown in each orchard and vegetable garden, but the nineteenth century, with the emergence of seed merchants, interrupted this continuity. These offered new cultivars which were the result of scientific and agronomic research dedicated to improved production. But in our changing world which cannot exist without constant dynamics of adaptation, evolution and even instability, the rediscovery of the smells, tastes and flavours of former times, which were all a complex alchemy of respect for the soil and for climatic cycles, and the effort to find old seeds which have been lost, is this not something of a vain search?

\section{INDEX}

Mots-clés : Potager, verger, variétés anciennes et locales, naturalité

Keywords : vegetable garden, orchard, ancient and local plant varieties, naturalness

\section{AUTEUR}

YVES-MARIE ALLAIN

Ingénieur horticole, paysagiste, Versailles ymallain@orange.fr 